\title{
A Short Review on Chitosan Membrane for Biomolecules Immobilization
}

\author{
Munusamy Thirumavalavan* and Jiunn-Fwu Lee* \\ Graduate Institute of Environmental Engineering, National Central University, Chung-Li, 32001, Taiwan, ROC \\ "Corresponding authors: Thirumavalavan M, Graduate Institute of Environmental Engineering, National Central University, Chung-Li, 32001, Taiwan, ROC, Tel: \\ +886-34279455; Fax: +886-34279455; E-mail: mtvala@yahoo.com
}

Jiunn-Fwu Lee, Graduate Institute of Environmental Engineering, National Central University, Chung-Li, 32001, Taiwan, ROC, Tel: +886-3-4227151-34658; Fax: +886-3-4226742; E-mail: jflee@ncuen.ncu.edu.tw

Received date: July 20, 2015, Accepted date: August 19, 2015, Published date: August 26, 2015

Copyright: (c) 2015 Thirumavalavan M, et al. This is an open-access article distributed under the terms of the Creative Commons Attribution License, which permits unrestricted use, distribution, and reproduction in any medium, provided the original author and source are credited.

\section{Abstract}

Chitosan is an important natural polymer that occurs in the world. Chitosan is soluble in acidic aqueous media and it can be obtained in various forms. It is widely used for many applications. Hence, we briefly describe the role of chitosan in membrane form, an important area of research in which a variety of synthetic methods has been proposed and their significance is explained. Thus, this review emphasizes the papers on the high value-added applications of these chitosan membranes in diverse fields such as medicine, biological, cosmetics and nanotechnology.

\section{Keywords Chitosan membrane; Biomolecules; Immobilization}

\section{Introduction}

The emerging field of biotechnology for the isolation or purification of biologically active molecules such as proteins, enzymes, peptides and nucleic acids requires a rapid development that emerges new technologies [1]. Such isolation or purification of biomolecules involves either adsorption or immobilization of biomolecules onto matrices that bearing functional groups [2]. Among these, the adsorption methods are considered to be ineffective due to weak bonding and low degree of stabilization [3]. So, the immobilization technique has received considerable attention as it provides several advantages including reuse, ease in separation, and improvement in stability. Such immobilization fixes the biomolecules onto solid inert support either by chemical adsorption (covalent bonding), van der waals force (physical adsorption) or encapsulation [3]. The selective and specific binding is based on affinity interaction between the support surface and biomolecules [4]. One of the most important aims of biotechnology is to enhance the conformational stability of the biomolecules and such stabilization is significantly controlled by the structure of biomolecules, the immobilization methods and the type of support. So, preparation of a suitable candidate for biomolecules immobilization has become thrust area of recent research. In general, the affinity column chromatography has been used for separation of biomolecules, but with limitations [1,5,6]. Recently, the affinity membrane has emerged as an alternative to column chromatography overcoming all the above said limitations $[1,5,6]$. The affinity membrane has provided some advantages over column chromatography such as higher flow rates, lower pressure drops, rapid binding and higher efficiency $[7,8]$. Actually, two approaches have been employed to prepare such affinity membranes. The most common method starts from commercially available microporous membranes such as polyethylene, polypropylene, nylon, poly(vinylidene difluoride), polysulfone or glass membranes. However, these membranes are usually hydrophobic and relatively inert. Thus, in order to overcome these drawbacks, a second approach was employed, in which membranes that contain functional groups such as cellulose membranes that contain $\mathrm{CH} 2 \mathrm{OH}$ and acetate functional groups were directly prepared. However, the cellulose membranes are also relatively hydrophobic, brittle, soluble in acids, and cannot easily acquire a macroporous structure. Hence they required some chemical modifications before they can be used. Later, the affinity membranes were either operated as macroporous membranes or fiber membranes $[9,10]$. In recent times, macroporous chitosan membranes with controlled pore size, good mechanical strength, chemical stability as well as biocompatibility have been developed $[1,5,6]$. Chitin and chitosan are natural polymers of remarkable availability and chitosan is a poly(2-amine-2-deoxy-Dglucose) which is obtained through poly( $\mathrm{N}$-acetyl-D-glucosamine) deacetylation of chitin in alkaline medium. Chitosan is an excellent material to produce membranes because of its hydrophilicity, reactivity, moldability, mechanical properties and biocompatibility. Hence chitosan membranes can be used in many areas such as food science, biochemistry, pharmaceuticals, medicine, agriculture and others [11]. Chitosan contains a large number of reactive hydroxyl $(\mathrm{OH})$ and amine (NH2) groups and consequently, it is no longer necessary to increase the number of these groups of the chitosan and these reactive groups can be readily modified using different ligands and also easily be coupled with biomolecules. Thus, chitosan can be an outstanding candidate for obtaining affinity membranes. Because of the presence of numerous amine groups in the chitosan molecules, the chitosan molecules can be cross-linked and the cross-linked chitosan membranes could also be employed for immobilization of biomolecules [1]. On the other hand, the choice of potential application of affinity membranes in the field of biotechnology is broad and thus there is a continuous quest for membrane materials which either possess some specific properties or have wide applicability which can easily be tailored for preferred properties.

\section{Characteristics and Significance of Chitosan}

Chitosan is actually obtained from chitin and when the degree of deacetylation of chitin reaches about $50 \%$ (depending on the origin of 
the polymer), it becomes soluble in aqueous acidic media and it is called as chitosan. Chitosan is a natural biodegradable polymeric material which is having good physical and biological properties. Chitosan is the only pseudonatural cationic polymer and thus, it finds many applications due to its unique characteristics. In the solid state, chitosan is actually a semicrystalline polymer. Its morphology has been investigated and many polymorphs are reported for chitosan in the literature [12].

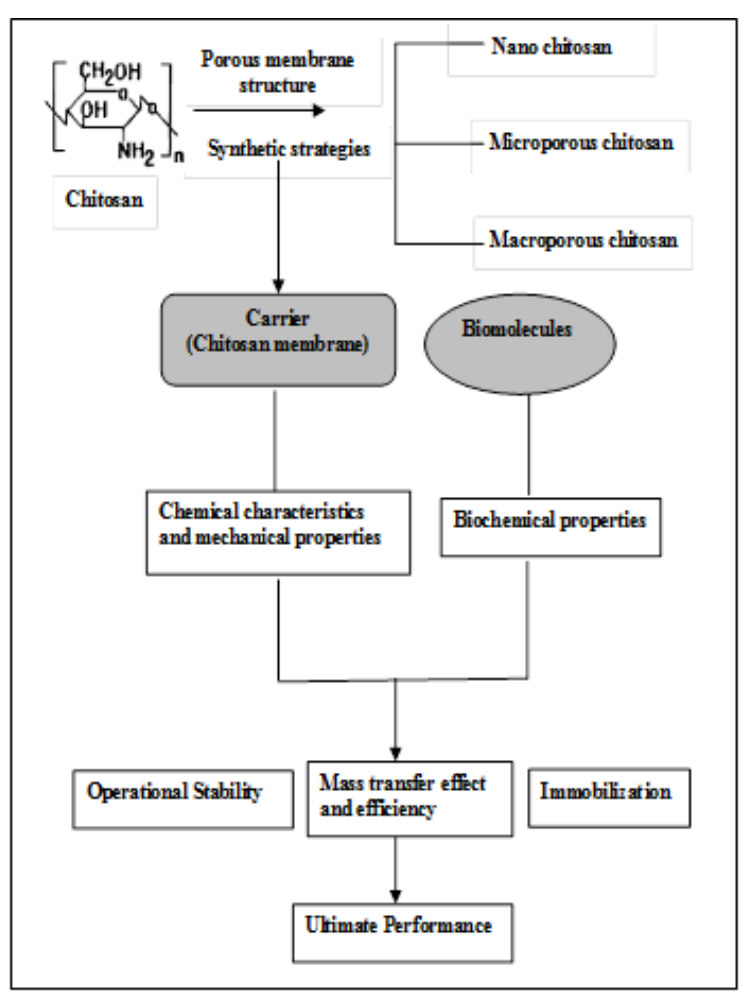

Figure 1: Schematic representation of characteristics of chitosan for immobilization.

The main exploration of chitosan concerns its preparation with varied molecular weights and deacetylation from chitin, the dependence of its solution properties, the preparation of its derivatives and its applications [12]. Chitosan can be obtained in various forms such as sponges, powders, membranes, beads and fibers [13]. The NH2 groups of the chitosan are involved in specific interactions with metals and thus chitosan is also known to have good complexing ability [12]. It is known that chitosan could enhance coagulation, suppress fibroblast growth and improve interstitial cell growth. It could also be used to prepare controlled-release medicine membranes, artificial skin and anti-adhesion membranes for post-operative applications [14]. Thus, chitosan meets several requirements in the biological, biotechnology or biomedical fields. The schematic representation of role and characteristics of chitosan membrane for immobilization is given in Figure 1.

\section{Immobilization of biomolecules}

The term immobilization refers to molecules physically confined or localized in a certain defined region with retention of their activities which really can be used repeatedly or continuously. In general, there are several reasons for the immobilization of biomolecules. The two main targeted benefits are easy separation of the biomolecules from the product and reuse of the biomolecules. Actually the ability of chitosan membrane to immobilize biomolecules, makes it possible to significantly expand the range of molecules that could be efficiently separated or purified [15].

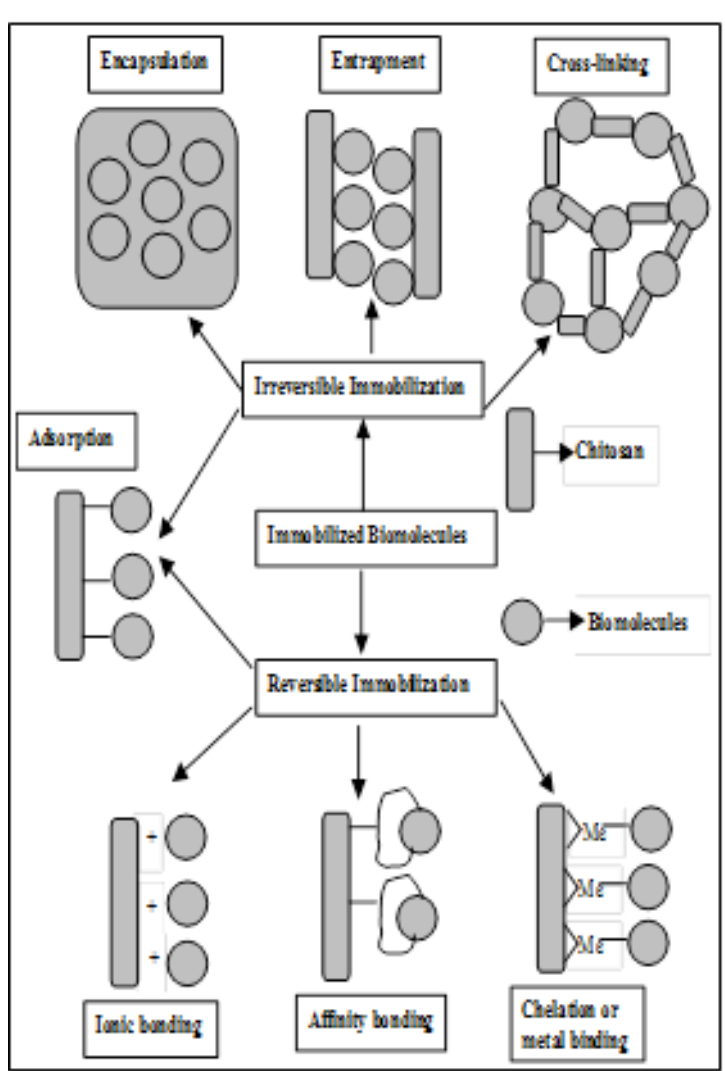

Figure 2: Schematic representation of possible modes of immobilization of biomolecules onto chitosan membrane.

The method of immobilization onto chitosan membrane can be divided into three main categories as follows;

1. Physical adsorption of biomolecules onto chitosan membrane.

2. Chemical adsorption of biomolecules onto chitosan membrane either directly or through a polymer or metal bridge.

3. Covalent, site-directed attachment of biomolecules directly to the chitosan membrane.

Physical absorption of biomolecules depends on the non-specific interaction of the biomolecules with the chitosan. These interactions occur due to ionic and hydrogen bonds, hydrophobic interactions, and van der Waals forces. Chemical adsorption of biomolecules can be obtained by covalent attachment of biomolecules with the functional group of the agents and it may be a non-site-directed-attachment. In such cases a variety of external agents such as polymer or metal bridges can be used. However covalent attachment of biomolecules can also be site-directed-attachment. The most effective means of sitedirected immobilization are those that bind the biomolecules via unique attachment sites directly to the chitosan. The immobilization of biomolecules may be either reversible or irreversible depending 
upon the conditions. Figure 2 schematically represents the possible modes of irreversible and reversible immobilization of biomolecules onto chitosan membrane.

\section{Chitosan Membranes Reported in Literatures}

Interestingly chitosan membranes have been reported for various applications such as for adsorption of heavy metals and immobilization of biomolecules as follows. Polyethersulfone supported microporous chitosan affinicity membrane was prepared by Zeng and Ruckenstein [1] in 1996 by phase inversion method for the adsorption of human serum albumin protein and the effect of temperature on the adsorption was studied. The results indicated that chitosan-Cibacron Blue F3GA affinity membrane exhibited enhanced adsorption of human serum albumin. Thus, by using this phase inversion method, they were only able prepare microporous chitosan membranes. Macroporous chitin membranes primarily were not able to be prepared, due to unavailability of suitable solvent and porogen. These macroporous chitosan membranes would definitely have relatively promising applications when compared with microporous membranes. Hence, in 1996, Zeng and Ruckenstein [5] proposed a novel method for the preparation of macroporous chitosan in which silica particles were employed as porogens as shown in Figure 3. The macroporous chitosan membrane was actually prepared by casting acidic chitosan solution containing silica particles, removing the solvent by evaporation, and finally dissolving the silica particles into an alkaline solution. This simple method resulted into chitosan membranes with high porosity and good mechanical strength. The pore size was significantly controlled by varying the size of silica particles. In order to prevent their dissolution in acidic solutions, the chitosan membranes were cross-linked using cross-linking agent (epichlorohydrin). In 1998, Zeng and Ruckenstein obtained macroporous chitosan membranes with controlled pore sizes and cross-linked with ethylene glycol diglycidyl ether to increase their chemical stability [6]. These chitosan membranes were used for adsorption of different protein molecules. The results obtained in this study clearly indicated that the cross-linked macroporous chitosan membranes could be used as efficient ion exchange membranes for protein separations.

In 2001, Ruckenstein and Guo [16] prepared mercerized macroporous cellulose membranes with large pore sizes and high porosity from filter paper by mercerization (caustic treatment) followed by cross-linking. These membranes were used for trypsin immobilization. The activities and stabilities of the immobilized trypsin membranes were investigated and compared [16]. Thus, it is very clear that macroporous chitosan membranes enhanced application of chitosan for both adsorption and immobilization phenomena.

$\mathrm{Hu}$ et al., in 2001 [14] have reported the preparation of chitosan membranes using fixation agents composed of alcohol, glycerol and potassium hydroxide and this sol-gel method significantly shortened the preparation time. The physical and biological properties were also studied. The results of this study clearly indicated that the addition of fixation agents could not only improve the flexibility of membranes but also improved the ability of wet keeping. This study revealed the effect of fixation agents on chitosan membrance characteristics during the process.

In 2004, Beppu et al., [17] have reported the preparation of histidine functionalized chitosan membrane for the removal of $\mathrm{Cu}^{2+}$ from aqueous solution. The results observed in this study indicated that the immobilization of histidine on porous chitosan membranes presented synergy with porosity and this synergy may be negative or positive, depending on the initial membrane porosity. Rashidova et al., in 2004 [18] have prepared hybrid nanocompositional chitosan/silica by solgel process through the interaction of organic polymers with tetraethoxysilane (TEOS). It was shown that the process consisted of two steps and properties of nanocomposite were mainly dependent on structure of the material.

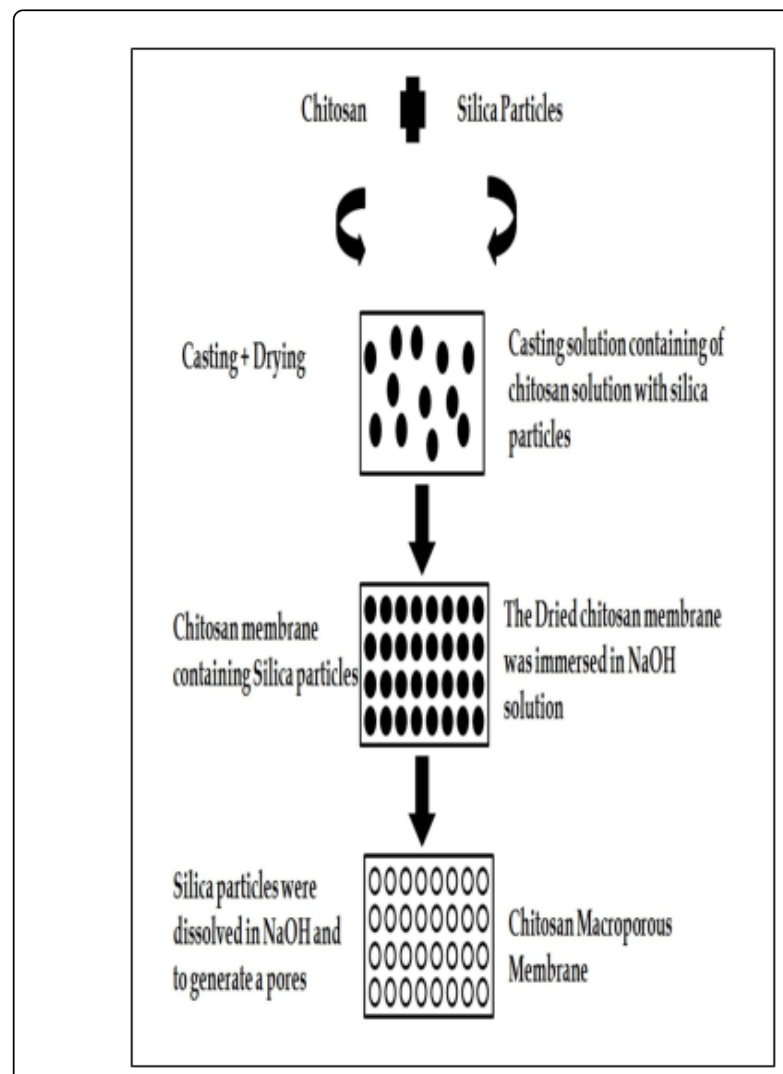

Figure 3: Preparation of macroporous chitosan membrane using silica particles.

In 2005, the adsorption of catalase was studied by Shentu et al., [2] using Cibacron Blue F3GA-attached chitosan microspheres (CB-CS). Significant amount of the adsorbed CAT (up to 90.6\%) was eluted in the elution medium and it revealed that CB-CS could be applied for adsorption of CAT without causing any denaturation.

In 2007, Beppu et al., [19] have studied the effect of cross-linked chitosan membrane on ion permeability and water adsorption and obtained the result that chemical modification with glutaraldehyde made chitosan more hydrophobic. The improvement in the properties of chitosan membranes was reported by Chen et al., in 2007 [20]. Chitosan based biomaterials were prepared using several organic acid solutions and the effect of these acids on dissolution of chitosan was also discussed by them. In their study, they concluded that the use of these organic acid solutions produced chitosan membranes with enhanced mechanical properties and hydrophlilicity for various biomedical applications. 
Adsoprtion and desoprtion of different metal ions were studied using cross-linked chitosan membrane by Laus et al., in 2010 [21]. For this purpose of cross-linking, both epichlorohydrin (ECH) and triphosphate (TPP) were used. The results obtained in this study suggested that the chitosan membranes could be effectively used in the processes of separation, preconcentration and uptake of metal ions from aqueous solutions. Reversible immobilization of catalase on polymer grafted and metal chelated chitosan membrane was reported by Bayramoglu and Arica, in 2010 [22]. The thermal stability of chitosan membrane was improved upon immobilization of catalase. It was also observed that the same support enzyme could be repeatedly used for immobilization of catalase after regeneration without significant loss in adsorption capacity or enzyme activity. In addition, polymer grafted and metal chelated chitosan membrane prepared in this work was considered to be a potential candidate for various biotechnological applications.

A novel L-lysine modified semi-crosslinked chitosan was synthesized by Dong et al., in 2011 [23] and it was demonstrated to be a promising enzyme support for the immobilization of glucose oxidases (GODs). Reports of this study indicated that the GODs immobilized chitosan membrane showed excellent operational stability and reusability. Also, the enzyme activity studied at a range of varied temperatures and $\mathrm{pH}$ indicated that the GODs immobilized onto chitosan had good thermal and $\mathrm{pH}$ stability.

Chitosan membranes were used for in vitro evaluation of bioactivity by Lieder et al., in 2013 [24]. They have recently developed a standardized and easily applied protocol for the solution casting of chitosan membranes displaying favorable bioactivity by sustaining cell attachment and proliferation for extended culture time. This protocol is appropriate for the use of chitosan materials and the investigation of chitosan derivatives with modified properties. Susanto et al. in 2013 [25] obtained chitosan based porous composite membrane with carbon nanotubes (CNTs) for immobilization of glucose oxidase and studied their potential use in biosensors. The results showed that enzyme immobilization capacity was greater for composite membranes and these membranes increased the stability of the enzyme immobilization. The immobilization of enzyme was influenced by both $\mathrm{pH}$ and the concentration of the enzyme solution. The presence of CNTs significantly enhanced the electrical conductivity of the chitosan membranes.

Recently our group has been actively working on macroporous chitosan membranes. We have developed a simple approach to prepare macroporous chitosan membranes using silica particles as porogens [26,27]. A variety of macroporous chitosan membranes was obtained by varying the particle size of silica porogens. The flow rate and characteristics of macroporous chitosan membranes were significantly affected by the particle size of silica porogens. We have also chemically modified the surface of macroporous chitosan membranes by cross-linking using various cross-linking agents. These cross-linked macroporous chitosan membranes were used for immobilization of various enzyme molecules. The immobilization and activity of enzymes were significantly enhanced by cross-linked macroporous chitosan membranes. We have also studied the various factors influencing both chitosan membrane characteristics and extent of immobilization. A versatile chitosan/ $\mathrm{ZnO}$ composite membranes was also successfully prepared by our group [28] using these macroporous chitosan membranes and nano $\mathrm{ZnO}$ obtained from tea extract by green synthesis method. This chitosan/ZnO composite membrane was also tested for its antimicrobial activities and indeed the chitosasn/ZnO composited showed enhanced antimicrobial activities when compared with chtiosan alone. The optical properties of both individual compounds and chitosan/ $\mathrm{ZnO}$ composite were also studied and the results confirmed the occurrence of intermolecular interaction between chitosan and $\mathrm{ZnO}$.

\section{Conclusions}

In this review we aimed to present an overview of the state of art in the knowledge and technical applications of chitosan membranes. An extensive bibliography of several studies has been included, which actually explains the synthesis of different chitosan membranes and their respective applications. The applications of both microporous and macroporous chitosan membranes are mainly discussed and based on the above studies, the macroporous chitosan membrane is considered to be effective candidate for both adsorption and immobilization techniques. Thus, this review in fact emphasizes the significance of macroporous chitosan membranes for the immobilization of various biomolecules. Nevertheless, this is an ambitious work and the very large number of studies published on a wide range of synthesis, properties and applications of chitosan membranes force us to make a selection from the most significant results obtained by the many groups including our team working around the world.

\section{References}

1. Zeng X, Ruckenstein E (1996) Supported chitosan-dye affinity membranes and their protein adsorption. J Membr Sci 117: 271-278.

2. Shentu J, Wu J, Song W, Jia Z (2005) Chitosan microspheres as immobilized dye affinity support for catalase adsorption. Int J Biol Macromol 37: 42-46.

3. Bautista FM, Bravo MC, Campelo JM, Garcia A, Luna D, et al. (1999) Covalent immobilization of acid phosphatase on amorphous AlPO4 support. J Mol Catal B: Enzymatic 6: 473-481.

4. Kawai T Saito K, Lee W (2003) Protein binding to polymer brush, based on ion-exchange, hydrophobic, and affinity interactions. J Chromatogr B Analyt Technol Biomed Life Sci 790: 131-142.

5. Zeng X, Ruckenstein E (1996) Control of pore sizes in macroporous chitosan and chitin membranes. Ind Eng Chem Res 35: 4169-4175.

6. Zeng X, Ruckenstein E (1998) Cross-linked macroporous chitosan anionexchange membranes for protein separations. J Membr Sci 148: 195-205.

7. Roper DK, Lightfoot EN (1995) Separation of biomolecules using adsorptive membranes. J Chromatogr A 702: 3-26.

8. Suen SY, Etzel MR (1992) A mathematical model of affinity membranes for bioseparations. Chem Eng Sci 47: 1355-1364.

9. Singh DK, Ray AR (1998) Characterization of grafted chitosn films. Carbohyd Polym 36: 251-255.

10. Okuyama K, Noguchi K, Miyazawa T, Yui T, Ogawa K (1997) Molecular and crystal structure of hydrated chitosan. Macromol 30: 5849-5855.

11. Beppu MM, Santana CC (2001) In vitro biomineralization of chitosan. Key Eng Mater 31: 192-195.

12. Rinaudo M (2006) Chitin and chitosan: Properties and applications. Prog Polym Sci 31: 603-632.

13. Krajewska B (2005) Membrane-based processes performed with use of chitin/chitosan materials. Sep Purifi Technol 41: 305-312.

14. Hu QL, Fang ZP, Zhao Y, Xu CW (2001) A new method to prepare chitosan membrane as a biomedical material. Chinese J Poly Sci 19: 467-470.

15. Nisnevitch M, Firer MA (2001) The solid phase in affinity chromatography: strategies for antibody attachment. J Biochem Biophys Methods 49: 467-480. 
Citation: Thirumavalavan M, Lee JF (2015) A Short Review on Chitosan Membrane for Biomolecules Immobilization. J Mol Genet Med 9: 178. doi:10.4172/1747-0862.1000178

Page 5 of 5

16. Ruckenstein E, Guo W (2001) Crosslinked mercerized cellulose membranes and their application to membrane affinity chromatography. J Membr Sci 187: 277-286.

17. Beppu MM, Arrud EJ, Vieira RS, Santos NN (2004) Adsorption of Cu(II) on porous chitosan membranes functionalized with histidine. J Membr Sci 240: 227-235.

18. Rashidova SSh, Shakarova DSh, Ruzimuradov ON, Satubaldieva DT, Zalyalieva SV, et al. (2004) Bionanocompositional chitosan-silica sorbent for liquid chromatography. J Chromatogr B Analyt Technol Biomed Life Sci 800: 49-53.

19. Beppu MM, Vieira RS, Aimoli CG, Santana CC (2007) Crosslinking of chitosan membranes using glutaraldehyde: Effect on ion permeability and water absorption. J Membr Sci 301: 126-130.

20. Chen PH, Hwang YH, Kuo TY, Liu FH, Lai JY, et al. (2007) Improvement in the properties of chitosan membranes using natura organic acid solutions as solvents for chitosan dissolution. J Med Biol Eng 27: 23-28.

21. Laus R Costa TG, Szpoganicz B, Fávere VT (2010) Adsorption and desorption of $\mathrm{Cu}(\mathrm{II}), \mathrm{Cd}(\mathrm{II})$ and $\mathrm{Pb}$ (II) ions using chitosan crosslinked with epichlorohydrin-triphosphate as the adsorbent. J Hazard Mater 183: 233-241.

22. Bayramoglu G, Arica MY (2010) Reversible immobilization of catalase on fibrous polymer grafted and metal chelated chitosan membrane. J Mol Catal B: Enzymatic 62: 297-304.
23. Dong LC, Wang G, Xiao Y, Xu Y, Zhou X, et al. (2011) Immobilization of glucose oxidase on a novel crosslinked chitosan support grafted with Llysine spacers. Chem Biochem Eng Q 25: 395-402.

24. Lieder R, Darai M, Orlygsson G, Sigurjonsson OE (2013) Solution casting of chitosan membranes for in vitro evaluation of bioactivity. Biol Proced Online 15: 11.

25. Susanto H, Samsudin AM, Rokhati N, Widiasa IN (2013) Immobilization of glucose oxidase on chitosan-based porous composite membranes and their potential use in biosensors. Enzyme Microb Technol 52: 386-392.

26. Yang WY, Thirumavalavan M, Malini M, Annadurai G, Lee JF (2014) Development of silica gel-supported modified macroporous chitosan membranes for enzyme immobilization and their characterization analyses. J Membr Biol 247: 549-559.

27. Yang WY, Thirumavalavan M, Lee JF (2015) Effects of porogen and cross-linking agents on improved properties of silica-supported macroporous chitosan membranes for enzyme immobilization. J Membr Biol 248: 231-240.

28. Malini M, Thirumavalavan M, Yang WY, Lee JF, Annadurai G (2015) A versatile chitosan/ $\mathrm{ZnO}$ nanocomposite with enhanced antimicrobial properties. Int J Biol Macromol 80: 121-129. 